\title{
The performances of eye drop instillation in glaucoma patients
}

\author{
Yuvaporn Tangseepha', Anita Manassakorn' \\ 'Department of Ophthalmology, Faculty of Medicine, Chulalongkorn University
}

\begin{abstract}
Aim: To evaluate the performances of the patients' eye drop instillation and estimate the quantity of eye drop needed per month in glaucoma patients.

Design: Cross-sectional, observational and questionnaire study

Methods: 137 glaucoma patients who had visual acuity better than 20/200 and had self-administered eye drops $\geq 6$ months were included. All patients were informed to apply artificial tears into their eyes. Performances were directly observed and evaluated according to the following criteria: washing hands before application, applying the drops into lower conjunctival fornix, successful instillation on the first attempt, did not contaminate the tip of the bottle with eye and adnexa, and occluded their nasolacrimal duct or closing of the eyelids after application. We also interviewed about the same tasks they always do at home. Nonparametric test were used for analyses.

Results: Median (IQR) age of the study population was 68 years $(18-89)$. Median (IQR) duration since diagnosis of glaucoma was 48 (6 - 576) months. During direct observation, only 1 patient (0.7\%) was able to accomplish all 5 criteria whereas 9 patients (6.6\%) could not accomplish any of the criteria. Twenty-nine patients (21.2\%) successfully instilled a drop in the lower fornix without touching the ocular adnexa. The overall performance under direct observation was significantly lower than the interview score $(p<0.001)$. Younger patients ( $<60$ years old) had higher performance under direct observation ( $p$ $=0.006$ ) and knew the correct techniques during interview session better than the older patients $(p=0.014)$. Fifty-eight patients $(42.4 \%)$ used more than 1 drop for each attempt. Number of eye drops used reported by the patients was significantly lower than what was directly observed $(p<0.001)$.

Discussion: Performance of self-administered eye drop was very poor. Age affected the ability of eye drop application. Standard technique should be emphasized to improve the performance of the glaucoma treatment and prevent contamination.
\end{abstract}

Key words: Antiglaucoma medications, Compliance, Eye drop, Glaucoma, Intraocular pressure

\section{Introduction}

Glaucoma is a chronic degenerative optic neuropathy which usually requires most patients to continue the use of antiglaucoma medications to control the intraocular pressure and prevent the progression of the disease. Patient compliance is the major key factor for successful treatment for glaucoma. Many investigators evaluated the adherence and persistence use of the medication via questionnaires,

Correspondence: Anita Manassakorn, Department of Ophthalmology, Chulalongkorn University and King Chulalongkorn Memorial Hospital, Thai Red Cross Society, Bangkok, Thailand, 10330.

E-mail:animanassa@gmail.com 
electronic monitoring devices and pharmacy claimed data. ${ }^{1-4}$ Aside from medication compliance, the perfect and aseptic techniques for eye drop applications are also important because ineffective administration will result in unsuccessful treatment and increase in the dosage which can cause ocular and systemic side effects, and therefore, non compliance. In addition, majority of our glaucoma patients were elderly and had difficulties administering the eye drops by themselves.

The number of drops used for each attempt needs to be considered. If the patient used more than 1 drop at a time, they will run out of the medication before the next visit. Many patients do not request for refill prior to their next appointment. As a result, the intraocular pressure will become high and the physicians will add more medications or seek surgical intervention to rectify the situation. Since medication is usually used as the initial management nowadays, the purpose of this study was to evaluate the performances of the patients' eye drop instillation and estimate the quantity of eye drops used per month.

\section{Materials and Methods}

This cross-sectional observational study and questionnaires was conducted at the Department of Ophthalmology, King Chulalongkorn Memorial Hospital, the Thai Red Cross Society, Bangkok, Thailand. We certify that all application institutional and governmental regulations concerning the ethical use of human volunteers were followed during this research. The protocol was reviewed and approved by the IRB of Chulalongkorn University, in concordance with the Declaration of Helsinki. All participants provided written informed consent to participate in the study.

The sample size was calculated using the following formula after a pilot study of 30 patients were completed.

$\begin{aligned} \mathrm{N} & = & \mathrm{Za^{2 } \mathrm { pq } / \mathrm { d } ^ { 2 }} \\ & = & 1.96^{2}(0.36)(0.64) / 0.08^{2} \\ & = & 138.3\end{aligned}$

One hundred and forty participants were diagnosed with glaucoma from outpatient clinics and treated with antiglaucoma medications. Patients who had visual acuity better than 20/200 and had self-administered the eye drops for at least 6 months were enrolled. Patients who had any neurological diseases or disability of the musculoskeletal system were excluded. Three patients were also excluded due to unable to perform visual field testing. One hundred and thirty-seven patients were informed to apply artificial tears $(2.5-\mathrm{ml}$ bottle) into their eyes under direct observation. We also instructed them to do the same procedures at home. An evaluation was performed according to the 5 following criteria: wash their hands before application, apply the drops into the lower conjunctival fornix, successful instillation on the first attempt, did not contaminate the tip of the eye drop bottle with the eye and/or adnexa, and perform nasolacrimal duct occlusion or close the eyelids after application. After that, one of the authors (YT) interviewed the patients about the methods they performed at home using the following questions. 
- Do you wash your hand before eye drop application?

- Do you apply eye drop in lower conjunctival fornix?

- Do you think your first drop land on ocular surface?

- Do you think the tip of eye drop did not contact with the eye and/or ocular adnexa?

- Do you perform nasolacrimal duct occlusion or close the eyelids after eye drop application?

The correlations for direct observation and interview scores vs. age group, eye laterality, gender, educational level, duration of glaucoma treatment, visual acuity and disease severity were then analyzed. The disease severity was classified by visual field mean deviation (MD) as mild (MD $\geq-6 \mathrm{~dB})$, moderate $(-12 \mathrm{~dB} \leq \mathrm{MD}$ $<-6 \mathrm{~dB}$ ), and severe (MD $<-12 \mathrm{~dB}$ ). Our success criteria was defined as using only 1 drop to land on the ocular surface without contamination to the ocular surface. In addition, we assessed the amount of eye drops needed for each attempt and calculated numbered needed per month.

\section{Statistical analyses}

All data were analyzed using SPSS software version 17 (SPSS Inc., Chicago, IL, USA). After the normality test was done, we used nonparametric method. Mann-Whitney $\mathrm{U}$ test was used for all comparison, except disease severity that was performed by Kruskal-Wallis test.

\section{Results}

One hundred and thirty-seven glaucoma patients were enrolled in the study. The demographic data of the study sample is shown in Table 1. Our study showed that under direct observation, only 1 patient $(0.7 \%)$ correctly applied the eye drop according to the 5 required criteria mentioned above whereas 9 patients $(6.6 \%)$ could not accomplish any of the criteria (Fig. 1).

Only 29 patients $(21.2 \%)$ accomplished the success criteria. According to the interview score, 22 patients (16.1\%) got full marks and 3 patients (2.2\%) did not know the correct techniques for eye drop application. The results of each application step and interview are shown in Figure 2.

The interview results showed that most of our patients knew the correct techniques, especially for washing their hands before application because 105 patients (75.6\%) reported performing this procedure before applying the medication (Fig. 2). However, only one-third of the patients knew that they had to apply the eye drop into the lower fornix. In addition, more than half of the patients contaminated the tip of the eye drop bottle by touching it to either the eye or adnexa. Hence, the performances of the eye drop instillation from the interview scores were statistically significantly higher than the observed scores $(P<0.001)$. The factors associated with the patients' performance for eye drop application are shown in Table 2. 
Table 1. Patient Baseline Characteristics

\begin{tabular}{|l|l|}
\hline Eyes (N) & 137 \\
\hline $\begin{array}{l}\text { Age, years } \\
\text { Median (IQR) }\end{array}$ & $68(13)$ \\
Range & $17-89$ \\
\hline $\begin{array}{l}\text { Gender, N (\%) } \\
\text { Male }\end{array}$ & $81(59)$ \\
Female & $56(41)$ \\
\hline $\begin{array}{l}\text { Education (highest degree), N (\%) } \\
\text { Below bachelor's degree }\end{array}$ & \\
Above bachelor's degree & $99(73.3)$ \\
\hline Duration of glaucoma, months & $38(26.7)$ \\
Median (IQR) & \\
Range & $48(72)$ \\
\hline Visual acuity, N (\%) & 6 to 576 \\
Equal or better than 20/40 & \\
Worse than 20/40 & $89(51.8)$ \\
\hline Glaucoma severity, N (\%) & $48(48.2)$ \\
\hline $\begin{array}{l}\text { Mild } \\
\text { Moderate }\end{array}$ & $45(37.5)$ \\
Severe & $37(30.8)$ \\
\hline
\end{tabular}

From the direct observation and interview scores, we found that patients younger than 60 years old performed better than the older group $(0.006$ and 0.014 , respectively). Other factors that can decrease the performance were not detected.

Fifty-eight patients (42.3\%) applied more than one drop at a time. To estimate the amount needed per month, we assumed that 1-drop was equivalent to 50 microliters. If a 3-ml eye drop was prescribed once daily at bedtime, that would mean that patients who used more than 2 drops a day will run out of medication before the next appointment. As a result of this, $20.0 \%$ of the patients will experience insufficient amount of medication. If a $5-\mathrm{ml}$ bottle was prescribed twice daily, 10 patients (7.4\%) will have the same problem before the next appointment (Table 3).

We also found that the number of eye drops used from the interview was significantly fewer compared to the direct observation $(P<0.001)$ and patients older than 60 years old usually used several drops compared to the younger individuals. $(P<0.001)$ 
Table 2. Summary of observation and interview scores

\begin{tabular}{|c|c|c|}
\hline Score & Observation & Interview \\
\hline & Median (points) & Median (points) \\
\hline \multicolumn{3}{|l|}{ Age } \\
\hline$\leq 60$ years & 3 & 4 \\
\hline$>60$ years & 2 & 3 \\
\hline Pvalue & 0.006 & 0.014 \\
\hline \multicolumn{3}{|l|}{ Gender } \\
\hline Male & 2 & 3 \\
\hline Female & 2 & 3 \\
\hline$P$ value & 0.704 & 0.574 \\
\hline \multicolumn{3}{|l|}{ Education } \\
\hline Below Bachelor's degree & 2 & 3 \\
\hline Bachelor's degree up & 3 & 4 \\
\hline Pvalue & 0.534 & 0.123 \\
\hline \multicolumn{3}{|l|}{ Duration } \\
\hline$<24$ months & 2 & 3 \\
\hline$\geq 24$ months & 2 & 3 \\
\hline Pvalue & 0.690 & 0.425 \\
\hline \multicolumn{3}{|l|}{ Visual acuity } \\
\hline Equal or better than $20 / 40$ & 2 & 3 \\
\hline Worse than $20 / 40$ & 2 & 3 \\
\hline Pvalue & 0.235 & 0.527 \\
\hline \multicolumn{3}{|l|}{ Glaucoma severity } \\
\hline Mild & 2 & 3 \\
\hline Moderate & 2 & 3 \\
\hline Severe & 2 & 3 \\
\hline Pvalue & 0.349 & 0.746 \\
\hline
\end{tabular}




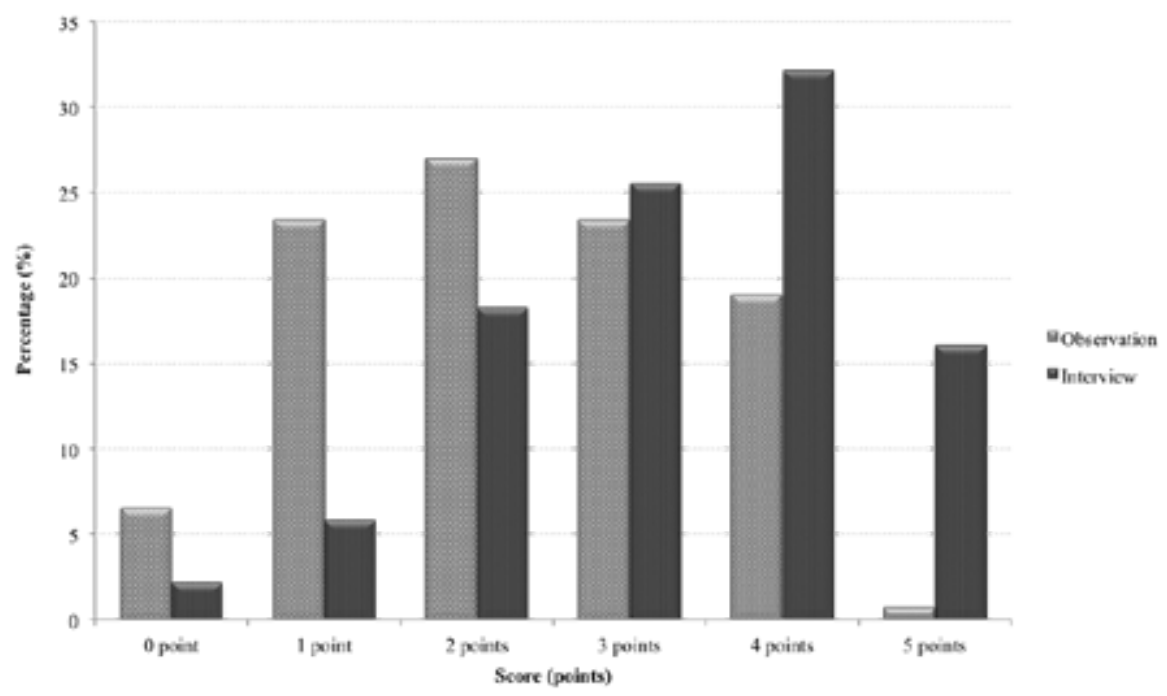

Fig 1. Performance of eye drop instillation according to observational score and interview score

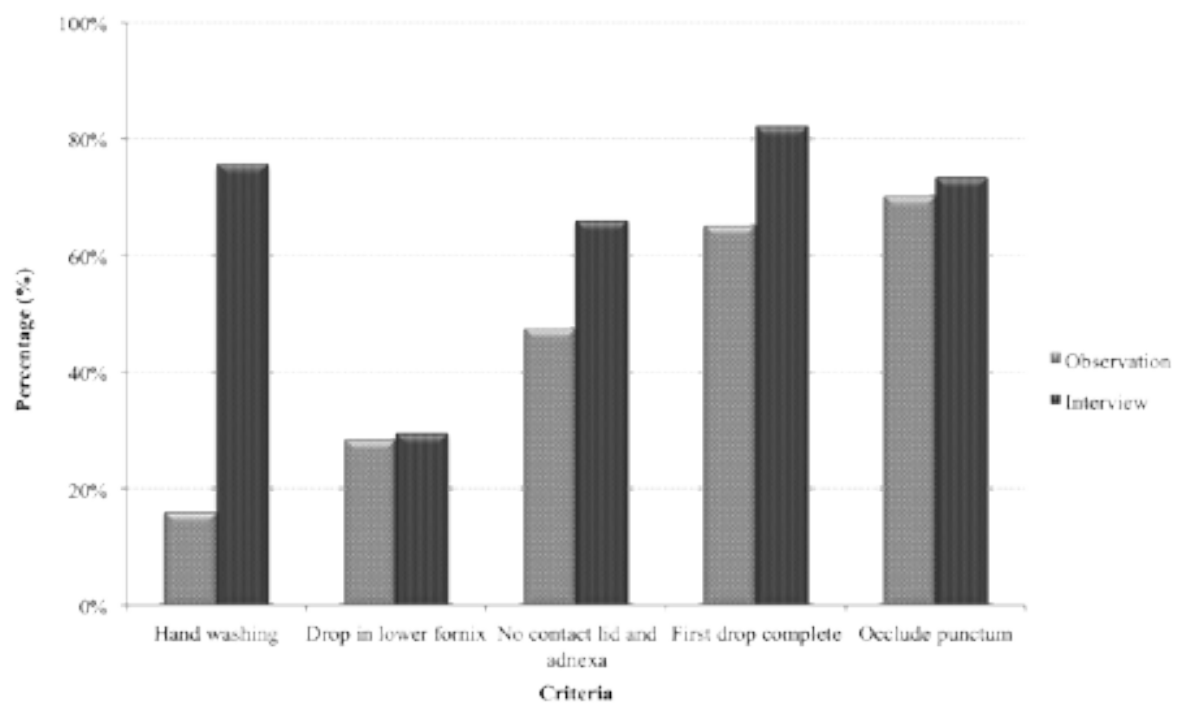

Fig 2. Distribution of observation and interview score 


\section{Discussion}

This study assessed the performances of eye drop instillation in glaucoma patients who self-administered the eye drop more than 6 months. The performance of eye drop application in our population was poor. Only 1 patient (0.7\%) accomplished the whole criteria and one-fifth success to put only 1 drop in the lower fornix without touching the ocular surface and adnexa. Older age was a risk factor for limited this performance. In addition, almost half of the patients used at least 2 drops for each attempt.

The performance of the eye drop instillation and magnitude of improper application were evaluated objectively and subjectively. ${ }^{5-8}$ Stone et al. ${ }^{7}$ used questionnaires and video recorders to evaluate the performance of the eye drop instillation with two different bottle sizes: $2.5-\mathrm{ml}$ and $15-\mathrm{ml}$ bottles. With 2.5 - $\mathrm{ml}$ bottle, $42.3 \%$ of our patients used at least 2 drops for each attempt compared to $22.4 \%$ of their study. According to the success criteria, we found $21.2 \%$ instilled only 1 eye drop into the eye without contamination that was similar to previous reports that ranged from $8.6 \%$ to $31.0 \% .5,7$ Another difference reported by the previous study was the risk factors that were associated with poorer performance such as the female gender and poor visibility ${ }^{7}$ which were not detected in our study. In contrast, previous study performed direct observation ${ }^{9}$ and found that young age was a factor for better performance, similar to ours.

Evaluation of eye drop instillation using questionnaires needed to be considered for falsely high performance. Previous study reported that the score for washing their hands and the contamination scores were $36.4 \%$ and $25.4 \%{ }^{6}$ compared to $16.1 \%$ and $47.4 \%$ in our findings. We found that the self-reported performances from interview sessions tended to overestimate the actual performance. Although, many of the patients claimed they have no difficulties in performing the eye drop instillation during interview sessions, the actual performance from the direct observation indicated otherwise. It is possible that the patients really did not realize that their technique is incorrect. Hence it is recommended to use direct observation to evaluate patient's performance. This indicated that the true number of patients who can properly perform the eye instillation process is much lower than what we expected. 
Table 3. Amount of eye drop needed at each attempt and percentage of patients who will experience insufficiency for $3-\mathrm{ml}$ and $5-\mathrm{ml}$ bottles.

\begin{tabular}{|c|c|c|c|c|}
\hline & $\begin{array}{l}\text { Drop } \\
\text { (median) }\end{array}$ & Range & $\begin{array}{l}\% \\
\text { Insufficient }\end{array}$ & \\
\hline & & & 3-ml bottle & 5-ml bottle \\
\hline Total & 1 & $1-10$ & 20 & 7 \\
\hline \multicolumn{5}{|l|}{ Age } \\
\hline$\leq 60$ years & 1 & $1-2$ & 0 & 0 \\
\hline$>60$ years & 1 & $1-10$ & 25 & 9 \\
\hline Pvalue & $<0.001$ & & & \\
\hline \multicolumn{5}{|l|}{ Gender } \\
\hline Male & 1 & $1-10$ & 19 & 9 \\
\hline Female & 1 & $1-4$ & 22 & 5 \\
\hline Pvalue & 0.523 & & & \\
\hline \multicolumn{5}{|l|}{ Education } \\
\hline Below Bachelor's degree & 1 & $1-10$ & 20 & 9 \\
\hline Bachelor's degree up & 1 & $1-4$ & 18 & 2 \\
\hline Pvalue & 0.158 & & & \\
\hline \multicolumn{5}{|l|}{ Duration } \\
\hline$<24$ months & 1 & $1-4$ & 26 & 5 \\
\hline$\geq 24$ months & 1 & $1-10$ & 17 & 8 \\
\hline Pvalue & 0.604 & & & \\
\hline \multicolumn{5}{|l|}{ Visual acuity } \\
\hline Equal or better than $20 / 40$ & 1 & $1-10$ & 19 & 7 \\
\hline Worse than $20 / 40$ & 1 & $1-10$ & 21 & 8 \\
\hline Pvalue & 0.758 & & & \\
\hline \multicolumn{5}{|l|}{ Glaucoma severity } \\
\hline Mild & 1 & $1-4$ & 18 & 4 \\
\hline Moderate & 1 & $1-10$ & 19 & 11 \\
\hline Severe & 1 & $1-4$ & 18 & 5 \\
\hline Pvalue & 0.856 & & & \\
\hline
\end{tabular}


Aside from the performance of the eye drop instillation, the number of drops used is equally important. In another direct observational study, it was reported that the patients used $1-8$ drops for each attempt. ${ }^{5}$ This indicated that the physician should always prescribe extra medication because it is unrealistic to assume that one drop will be accomplished per attempt or instruct the patients to come in for a refill when they run out of medication regardless of their next appointment. Although the results from previous studies varied due to the different settings, we can imply that the performance of eye drop application was poor worldwide. As a result of this, it is even more pertinent that physicians should assess the proper eye instillation process before assuming the dosage and/or efficacy of the medication need to be adjusted or changed.

There were some limitations in our study. We used artificial tear eye drops so the size and shape of the bottles varied which could have affected the application process. However, we selected bottles composed of the same material to avoid differences in the pressure that is used to apply the medication. Second, the setting during direct observation such as the lighting and having adequate space to move in may not be the same as the patients' home which could have altered their performances. Lastly, we did not explore adherence, persistence, handling and storage of the medication in this study. Additional larger study incorporating all of these issues mentioned above is warranted.

In conclusion, our findings were consistent with previous reports that even in experienced patients, proper eye drop instillation was poor. We recommend that training or retraining patients and their relatives is necessary to improve this task. Not only for glaucoma management and prevention of side effects but it will also prevent unnecessary expenses. Physicians need to be aware that extra quantity of the medication should be incorporated into the dosage calculation, especially for the elderly patients.

\section{References}

1. Okeke CO, Quigley HA, Jampel HD, Ying GS, Plyler RJ, Jiang, Y, et al. Adherence with topical glaucoma medication monitored electronically the Travatan Dosing Aid study. Ophthalmology. 2009;116(2):191-199.

2. Hermann MM, Bron AM, Creuzot-Garcher CP, Diestelhorst, M. Measurement of Adherence to Brimonidine Therapy for Glaucoma Using Electronic Monitoring. J Glaucoma. 2011;20(8):502-508.

3. Reardon G, Kotak S, Schwartz GF. Objective assessment of compliance and persistence among patients treated for glaucoma and ocular hypertension: a systematic review. Patient Prefer Adher. 2011;5:441-463.

4. Sleath B, Robin AL, Covert D, Byrd JE, Tudor G, Svarstad B. Patient-reported behavior and problems in using glaucoma medications. Ophthalmology. 2006;113(3):431-436.

5. Gupta R, Patil B, Shah BM, Bali SJ, Mishra SK, Dada T. Evaluating eye drop instillation technique in glaucoma patients. J Glaucoma. 2012;21(3):189-192.

6. Tsai T, Robin AL, Smith JP. An Evaluation of How Glaucoma Patients Use Topical Medications: A Pilot Study. Trans Am Ophthalmol Soc. 2007;105:7.

7. Stone JL, Robin AL, Novack GD, Covert DW, Cagle GD. An objective evaluation of eyedrop instillation in patients with glaucoma. Arch Ophthalmol. 2009;127(6):732-736. 
8. Hennessy AL, Katz J, Covert D, Protzko C, Robin AL. Videotaped evaluation of eyedrop instillation in glaucoma patients with visual impairment or moderate to severe visual field loss. Ophthalmology. 2010;117(12):2345-2352.

9. Aptel F, Masset H, Burillon C, Robin A, Denis P. The influence of disease severity on quality of eye-drop administration in patients with glaucoma or ocular hypertension. Br J Ophthalmol. 2009;93(5):700-701. 